\title{
Differential susceptibility to food stress in neonates of sexual and asexual mollies (Poecilia, Poeciliidae)
}

Tobler, Michael ; Schlupp, Ingo

\begin{abstract}
The maintenance of sex is still an evolutionary puzzle given its immediate costs. Stably coexisting complexes of asexually and sexually reproducing forms allow to study mechanisms that balance the costs and benefits of both asexual and sexual reproduction. Here, we tested whether coexisting asexual and sexual fish of the genus Poecilia differed in neonate mortality when exposed to environmental stress in the form of fluctuating temperatures and food deprivation. We find that asexual Amazon mollies, Poecilia formosa, are significantly more sensitive to food stress than their sexual relative Poecilia latipinna, but both are equally unaffected by variable temperatures. Differences in the susceptibility to environmental stress may contribute to diminishing the asexuals' benefits of a higher intrinsic population growth rate and thus mediate stable coexistence of the two reproductive forms
\end{abstract}

DOI: https://doi.org/10.1007/s10682-008-9288-7

Posted at the Zurich Open Repository and Archive, University of Zurich ZORA URL: https://doi.org/10.5167/uzh-156306

Journal Article

Published Version

Originally published at:

Tobler, Michael; Schlupp, Ingo (2010). Differential susceptibility to food stress in neonates of sexual and asexual mollies (Poecilia, Poeciliidae). Evolutionary Ecology, 24(1):39-47.

DOI: https://doi.org/10.1007/s10682-008-9288-7 


\title{
Differential susceptibility to food stress in neonates of sexual and asexual mollies (Poecilia, Poeciliidae)
}

\author{
Michael Tobler $\cdot$ Ingo Schlupp
}

Received: 13 March 2008/Accepted: 26 November 2008/Published online: 11 December 2008

(C) Springer Science+Business Media B.V. 2008

\begin{abstract}
The maintenance of sex is still an evolutionary puzzle given its immediate costs. Stably coexisting complexes of asexually and sexually reproducing forms allow to study mechanisms that balance the costs and benefits of both asexual and sexual reproduction. Here, we tested whether coexisting asexual and sexual fish of the genus Poecilia differed in neonate mortality when exposed to environmental stress in the form of fluctuating temperatures and food deprivation. We find that asexual Amazon mollies, Poecilia formosa, are significantly more sensitive to food stress than their sexual relative Poecilia latipinna, but both are equally unaffected by variable temperatures. Differences in the susceptibility to environmental stress may contribute to diminishing the asexuals' benefits of a higher intrinsic population growth rate and thus mediate stable coexistence of the two reproductive forms.
\end{abstract}

Keywords Asexuality · Evolution and maintenance of sex · Gynogenesis · Environmental stress · Mutation accumulation

\section{Introduction}

The prevalence and maintenance of sexual reproduction in the light of its costs is still an unresolved problem in evolutionary biology (Maynard Smith 1978; Kondrashov 1993; West et al. 1999). Sexual reproduction is costly in two ways (Maynard Smith 1978; Bell 1982): (1) Sexual species need to produce dispensable males. At a sex ratio of 1:1, this

M. Tobler $(\bowtie)$

Institute of Zoology, University of Zürich, Winterthurerstr. 190, CH-8057 Zürich, Switzerland e-mail: michi.tobler@gmail.com

M. Tobler · I. Schlupp

Department of Zoology, University of Oklahoma, 730 Van Vleet Oval, Norman, OK 73019, USA

Present Address:

M. Tobler

Department of Biology and Department of Wildlife and Fisheries Sciences, Texas A\&M University, 2258 TAMU, College Station, TX 77843-2358, USA 
essentially results in a twofold lower population growth rate compared to similar asexually reproducing organisms ('twofold cost of sex'). (2) As an effect of meiosis and recombination, sexual organisms only pass half of their genes to the next generation. Furthermore, successful genotypes are destroyed in every reproductive cycle. Consequently, asexual lineages that avoid the costs of sex have an advantage over related sexual forms and-at least under the assumption of all other things being equal-should out-compete them over time (Maynard Smith 1978; Bell 1982; Lively and Lloyd 1990; Ladle 1992; Barton and Charlesworth 1998).

Sperm-dependent asexuals (gynogens) are powerful model systems to study the costs and benefits of sex. Gynogens require sperm of closely related sexual species to trigger embryogenesis, but inheritance is strictly clonal, i.e., without paternal contribution (Dawley 1989; Vrijenhoek 1994; Schlupp 2005). Due to their sperm dependence, gynogens have to coexist with closely related sexual species (Niemeitz et al. 2002; Choleva et al. 2008). Stable coexistence of sexually and asexually reproducing forms, however, is paradoxical if the costs of sex are considered: gynogens should outcompete their sexual relatives due to the twofold advantage in population growth rate, just to go extinct themselves after the disappearance of their sexual sperm donors (Schlupp 2005; Kokko et al. 2008). Nonetheless, gynogenetic complexes persist in natural systems; the costs of sex must thus be balanced by some benefits.

A diverse set of mechanisms has previously been discussed to select for sex (see Kondrashov 1993 for an overview). Due to the lack of recombination, asexuals have been proposed to have a lower evolvability. For example, sexual organisms are more efficient in purging deleterious mutations, and beneficial mutations which may arise independently in different individuals can be combined via recombination. Asexual organisms, on the other hand, cannot avoid accumulating deleterious mutations, which eventually will impose fitness costs and may lead to the extinction of a lineage (Kondrashov 1982, 1988; Charlesworth 1990; Barton and Charlesworth 1998). Accumulation of deleterious mutations for instance may lead to a higher susceptibility to environmental stress (Vrijenhoek and Pfeiler 1997; Lively et al. 1998). Lower evolvability may also impose short-term fitness costs. For example, asexuals are thought to be disproportionally more susceptible to rapidly coevolving parasites, an idea that has been popularized as the 'Red Queen hypothesis' (Hamilton 1980; Lively 1989; Hamilton et al. 1990; Lively et al. 1990; but see Salathé et al. 2008; Tobler and Schlupp 2008). In gynogenetic complexes, where asexuals rely on heterospecific sperm for reproduction, avoidance of gynogens by sperm donors could also lead to the reduction of the twofold benefit of asexuality (Hubbs 1964; Ryan et al. 1996; Riesch et al. 2008).

We studied a gynogenetic fish, the Amazon molly, Poecilia formosa (Girard 1859), and its sexual sperm donor, the Sailfin molly, Poecilia latipinna (LeSueur 1821), to ask whether differential susceptibility of asexuals to environmental stress may contribute to the stability of the gynogenetic complex. At least in the laboratory, asexual and sexual mollies have the same reproductive output (Schlupp and Tobler, unpublished data), thus $P$. formosa should theoretically be able to out-compete their sexual relatives. Since mixed populations appear to be stable in the field (Schlupp 2005), the basic assumption of the theory cannot be fulfilled. Obviously, not all other things are equal between asexual and sexual mollies, and $P$. formosa must suffer from some fitness reductions to stably coexist with their sperm donors. In this study, we tested whether asexual $P$. formosa are more susceptible to environmental stress than sexual $P$. latipinna. We compared neonate survival in a laboratory experiment during which we exposed asexual and sexual mollies to different temperature and food regimes. 


\title{
Materials and methods
}

\author{
Breeding fish
}

Poecilia formosa and P. latipinna females were collected at Lincoln Park in Brownsville, Texas, USA (N25.900 ${ }^{\circ}$ W97.480 $)$ in May 2005 and transported to a greenhouse of the Aquatic Research Facility at the University of Oklahoma. Fish were housed individually in plastic tanks $(4$ l) covered with mesh wire to prevent escaping. A flow-through system using untreated well water was employed to maintain proper water quality. Naturally growing algae and mosquito larvae served as food basis, which was supplemented with commercially available flake food three times a week.

Since mollies can store sperm (Constantz 1989), no males were added to the females. Offspring born in the setup were thus sired by males from the natural populations. All females' tanks were checked for offspring daily. Ten $P$. formosa females and thirteen $P$. latipinna females gave birth in the course of the experiment. Neonates were immediately removed and they entered the experiment (see below) or were transferred to stock tanks.

\section{Experimental setup}

During the experiment, neonates were individually housed in 2-1 tanks. Each tank was aerated and connected to a flow-through system using untreated well water, so that the total water volume was replaced about every second day. Furthermore, each tank was equipped with a Visi-Therm $25 \mathrm{~W}$ aquarium heater. In the fish room, temperature was held constant at $18^{\circ} \mathrm{C}$.

Four siblings were randomly chosen from each brood and randomly assigned to one of four treatment groups. We used a $2 \times 2$ fully factorial design manipulating food availability (high vs. low) and temperature regime (constant vs. variable).

Temperature. In the stable temperature treatment, the thermostat of the heater was set at a constant temperature of $23^{\circ} \mathrm{C}$. In the variable temperature treatment, the thermostat was set to $28^{\circ} \mathrm{C}$, and heaters were connected to a timer switching the heater off and on every $4 \mathrm{~h}$. This resulted in a temperature fluctuating between 18 and $28^{\circ} \mathrm{C}$ over $8 \mathrm{~h}$. Temperature was monitored over 28 days in six randomly chosen tanks of each temperature treatments using $\mathrm{HOBO}$ v2 pro data loggers (Onset Computer Corporation, Bourne, MA).

Food. Fine grained commercially available fish food was used to feed the fish. In the high food treatment, $1 \mathrm{mg}$ of food was provided on a daily basis. In the low food treatment, $1 \mathrm{mg}$ food was only provided every other day.

As a response variable, we recorded mortality in our experimental setup every morning. The experimental trials were terminated after 28 days and fish that survived up to that time were returned to stock tanks. Experimental data were analyzed using a survival analysis (Cox regression). 'Age at death' was used as dependent variable. Individuals still alive at the end of the experiment were censored. 'Species', 'food treatment', 'temperature treatment', as well as all two-way interactions were used as independent variables. SPSS 16 (SPSS Inc., Chicago, IL) was used for all statistical analyses.

Size at birth might affect the tolerance to environmental stress of neonates. Since neonates are highly susceptible to handling stress (Tobler, personal observation), fish that were used in the experiment were not measured. To exclude the possibility that size differences between $P$. latipinna and $P$. formosa at birth affected our results, we measured the standard length of four randomly chosen siblings of the individuals used in the 
experiment utilizing digital photographs of each individual. Measurements were then taken using the Spot Advanced 4.5 computer software (Diagnostic Instruments Inc., Sterling Heights, MI).

\section{Results}

In the constant temperature treatment, temperature was $23.2 \pm 1.8^{\circ} \mathrm{C}(N=6$ tanks $)$ and in the variable temperature treatment $22.7 \pm 6.1^{\circ} \mathrm{C}(N=6$ tanks $)$ confirming that there was no significant difference in the mean (independent sample $t$-test: $t_{10}=0.193, P=0.851$ ) but a significant difference in the degree of the fluctuations ( $F$-test for equal variances: $\left.F_{5,5}=11.485, P=0.009\right)$.

The experiment revealed that there was a significant interaction effect between species and food treatment on the survival of newborn mollies (Tables 1, 2). Asexual P. formosa had an eminently low life expectancy in the low food treatment (Fig. 1). The temperature treatment did not have a significant effect on the survival of neonates. It is unlikely that the higher susceptibility to food deprivation in $P$. formosa is caused by a size difference of mollies at birth. The size at birth did not differ significantly between sexual and asexual mollies (P. latipinna, SL $9.8 \pm 1.1 \mathrm{~mm}, N=13$ clutches; $P$. formosa, SL $9.2 \pm 0.9 \mathrm{~mm}$, $N=10$ clutches; independent sample $t$-test: $t_{21}=1.400, P=0.176$ ).

Table 1 Mean \pm SE survival time in days as well as 95\% confidence intervals (lower bound, upper bound) in neonates of the sexual $P$. latipinna $(N=13$ in each treatment) and the asexual $P$. formosa $(N=10$ in each treatment) exposed to different environmental conditions. Descriptive statistics were calculated using a Kaplan-Meier analysis with censoring

\begin{tabular}{lllll}
\hline & $\begin{array}{l}\text { Stable } \mathrm{T} \\
\text { High food }\end{array}$ & $\begin{array}{l}\text { Variable } \mathrm{T} \\
\text { High food }\end{array}$ & $\begin{array}{l}\text { Stable T } \\
\text { Low food }\end{array}$ & $\begin{array}{l}\text { Variable T } \\
\text { Low food }\end{array}$ \\
\hline P. formosa & $23.6 \pm 1.6$ & $19.7 \pm 2.4$ & $13.7 \pm 1.5$ & $14.5 \pm 2.0$ \\
& $(20.4,26.8)$ & $(15.1,24.3)$ & $(10.7,16.7)$ & $(10.6,18.4)$ \\
P. latipinna & $17.5 \pm 1.9$ & $20.1 \pm 1.6$ & $18.2 \pm 2.5$ & $16.6 \pm 2.0$ \\
& $(13.8,21.3)$ & $(16.9,23.2)$ & $(13.3,23.2)$ & $(12.6,20.6)$ \\
\hline
\end{tabular}

Table 2 Survival analysis (Cox regression) of neonates in the experiment. Significant effects are in bold face. $-2 \log$ likelihood $=592.435 ; \chi^{2}=16.526 ; d f=6, P=0.011$

\begin{tabular}{|c|c|c|c|c|c|c|c|c|}
\hline \multirow[t]{2}{*}{ Factor } & \multirow[t]{2}{*}{ B } & \multirow[t]{2}{*}{ SE } & \multirow[t]{2}{*}{ Wald } & \multirow[t]{2}{*}{$d f$} & \multirow[t]{2}{*}{$P$} & \multirow[t]{2}{*}{$\operatorname{Exp}(\mathrm{B})$} & \multicolumn{2}{|c|}{$95 \%$ CI for $\operatorname{Exp}(\mathrm{B})$} \\
\hline & & & & & & & Lower & Upper \\
\hline Species & 0.527 & 0.434 & 1.473 & 1 & 0.225 & 1.693 & 0.723 & 3.963 \\
\hline Temperature & 0.212 & 0.450 & 0.223 & 1 & 0.637 & 1.236 & 0.512 & 2.985 \\
\hline Food & 1.385 & 0.443 & 9.781 & 1 & 0.002 & 3.993 & 1.677 & 9.509 \\
\hline Species $\times$ temperature & -0.017 & 0.469 & 0.001 & 1 & 0.972 & 0.983 & 0.983 & 2.466 \\
\hline Species $\times$ food & -1.286 & 0.481 & 7.162 & 1 & 0.007 & 0.276 & 0.108 & 0.709 \\
\hline Temperature $\times$ food & -0.066 & 0.466 & 0.020 & 1 & 0.888 & 0.936 & 0.375 & 2.335 \\
\hline
\end{tabular}



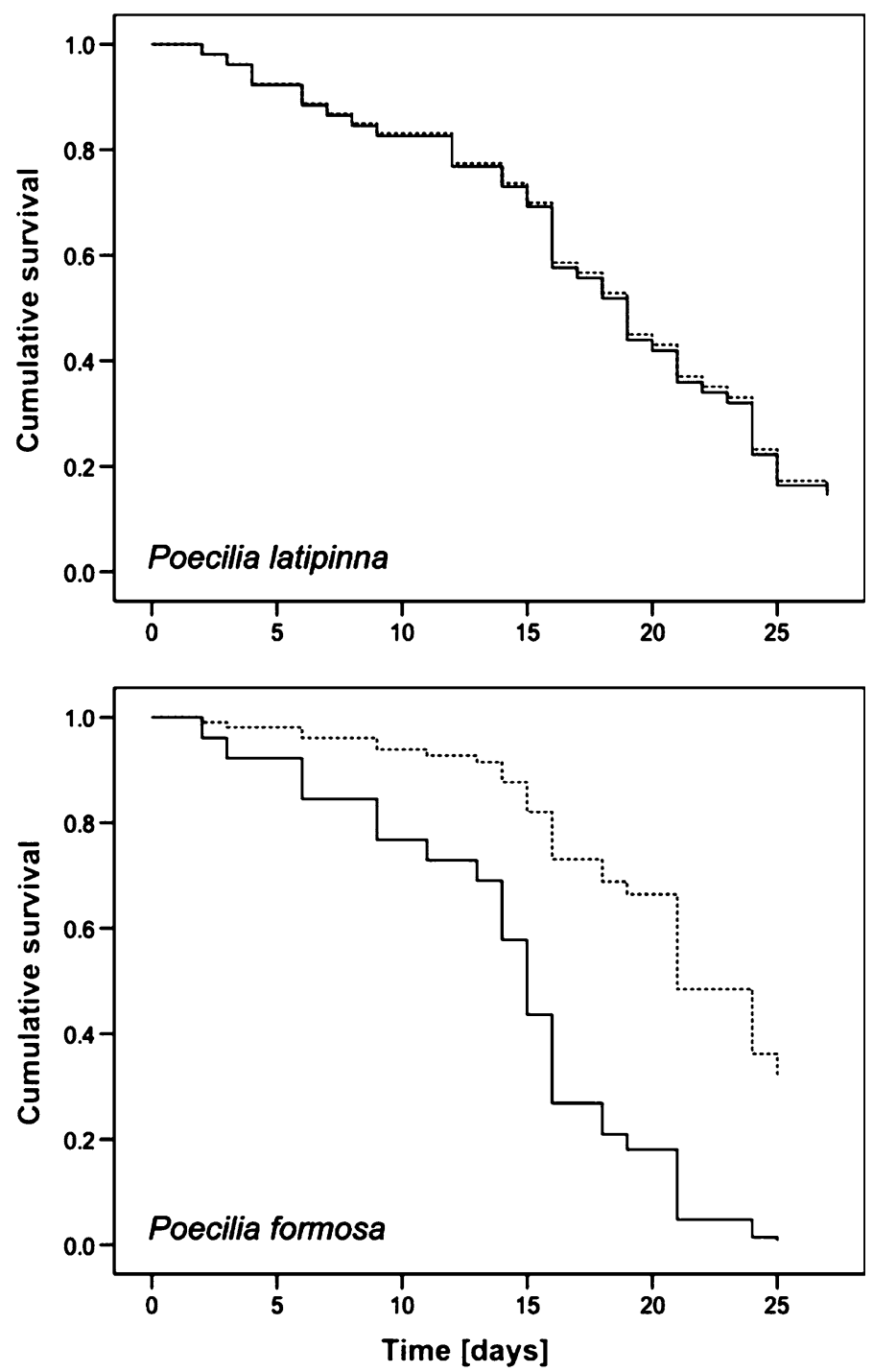

Fig. 1 Survival plots showing the cumulative survival of sexual P. latipinna and asexual $P$. formosa under the high $(\cdots)$ and low $(-)$ food treatment

\section{Discussion}

We detected a significant interaction effect between the factors 'species' and 'food treatment' in our experiment indicating that asexual $P$. formosa performed worse under food stress. Increased juvenile mortality due to resource limitation may be one mechanism contributing to diminishing the twofold advantage asexual mollies have in terms of their intrinsic population growth rate and might thus mediate the stable coexistence of asexual and sexual reproductive forms. 
Differential susceptibility to environmental stress has also been shown to play a role in the coexistence between asexual and sexual fish of the genus Poeciliopsis (Vrijenhoek and Pfeiler 1997). Sexuals and two distinct clones have been found to differ in their susceptibility to cold and heat stress as well as hypoxia. The hypothesis of differential susceptibility to environmental stress was also tested-but not confirmed-in the asexuals and sexuals of the freshwater snail Potamopyrgus antipodarum (Lively et al. 1998).

Two not mutually exclusive mechanisms may cause the differential susceptibility to food stress in asexual $P$. formosa. Firstly, increased susceptibility to stress may be caused by the accumulation of deleterious mutations (Wolfe 1993; Kondrashov and Houle 1994; Cooper et al. 2005; Buckling et al. 2006; Killick et al. 2006), which asexuals accumulate over time due to the lack of recombination (Kondrashov 1982, 1988; Charlesworth 1990). Poecilia formosa presumably originated through a single natural hybridization event (between P. latipinna and P. mexicana) about 120,000 generations ago (Schartl et al. 1995; Lampert et al. 2005; Lampert and Schartl 2008). This is old under mutation accumulation models, and effects of deleterious mutations would be expected (Gabriel et al. 1993; Gabriel and Bürger 2000). In fact, a recent study applying a simple model of mutation accumulation found that extinction of $P$. formosa would be expected to occur within a timeframe that is less than the actual age of the species (Loewe and Lamatsch 2008). Hence, the differential susceptibility to food stress documented here could be a manifestation of mutation accumulation in asexual lineages. Further investigations using triploid $P$. formosa could potentially establish a connection between deleterious mutations and stress susceptibility. Triploid $P$. formosa evolved relatively recently in the Río Purificación and Río Guayalejo river drainages in Mexico, whereby an additional set of $P$. mexicana chromosomes was incorporated to the old hybrid genome (Balsano et al. 1989; Lampert et al. 2005; Schories et al. 2007). The addition of an extra set of chromosomes would be expected to at least partly alleviate the effects of accumulated deleterious mutations in the asexual hybrid genome. Hence, if deleterious mutations indeed are causing the increased susceptibility to food stress in diploid P. formosa, we would expect a higher stress resistance in triploids.

Secondly, asexuals have been hypothesized to freeze and replicate ecologically relevant genetic variation that segregates in their sexual ancestors (Vrijenhoek 1979; Wetherington et al. 1989; Semlitsch et al. 1997). Susceptibility to environmental stressors may thus be directly inherited by the distant sexual ancestors (e.g., in asexual fish of the genus Poeciliopsis, Vrijenhoek and Pfeiler 1997). In this case, stress susceptibility within each clone of $P$. formosa should represent only a fraction of the total variation in stress susceptibility observed in the sexual ancestors ( $P$. latipinna and $P$. mexicana). However, the high age combined with the single origin of $P$. formosa precludes a rigorous test of this hypothesis as variation in traits of the parental populations may have changed in the 120,000 generations since the original hybridization occurred.

The differential susceptibility to food stress documented here in combination with seasonal variation in food availability may ultimately contribute to mediating stable coexistence of sexuals and asexuals. Although exposed to identical environmental conditions (Schlupp and Ryan 1996), asexual P. formosa significantly increase in relative frequency over the summer, presumably due to the higher population growth rate, but decrease during the cold and less productive winter months (Heubel 2004). Further investigations will have to show how seasonal changes in food availability affect the fitness of $P$. formosa in natural habitats, and how susceptibility to environmental stress varies across different life stages in mollies. 
Research to date has not been able to single out one mechanism explaining the balance of cost and benefits of asexual and sexual reproductive strategies in the P. formosa system. To date, differences in susceptibility to parasites (Tobler and Schlupp 2005; Tobler et al. 2005) and to environmental stress (Heubel 2004 and this study) as well as behavioral regulation through male mate choice of sexual sperm donors (see Schlupp 2005 for a review) have been considered. Future efforts need to focus on potential interaction effects between different mechanisms diminishing the twofold advantage of asexuals. Although 'a beautiful phenomenon as sex deserves a nice, simple explanation' (Kondrashov 1999), it seems that multiple selective forces and their interactions need to be considered to explain the stability of coexisting asexuals and sexuals in specific cases (West et al. 1999).

Acknowledgments We thank Tami Thomason and Wendal Porter for technical support. This research project was approved by the Animal Care and Use Committee of the University of Oklahoma (AUS R05014). Texas Parks \& Wildlife issued the permit to collect fish (SPR-0305-045). Financial support came from the Basler Foundation for Biological Research, the Janggen-Poehn-Foundation, the Roche Research Foundation, and the Wolfermann-Nägeli-Foundation (to M.T.) as well as the University of Oklahoma Faculty Senate (to I.S.).

\section{References}

Balsano JS, Rasch EM, Monaco PJ (1989) The evolutionary ecology of Poecilia formosa and its triploid associate. In: Meffe GK, Snelson FF (eds) Ecology and evolution of lifebearing fishes (Poeciliidae). Prentice Hall, New Jersey, pp 277-297

Barton NH, Charlesworth B (1998) Why sex and recombination? Science 281:1986-1990. doi:10.1126/ science. 281.5385 .1986

Bell G (1982) The masterpiece of nature, the evolution and genetics of sexuality. University of California Press, Berkeley

Buckling A, Wei Y, Massey RC, Brockhurst MA, Hochberg ME (2006) Antagonistic coevolution with parasites increases the cost of host deleterious mutations. Proc R Soc B Biol Sci 273:45-49. doi:10.1098/rspb.2005.3279

Charlesworth B (1990) Mutation-selection balance and the evolutionary advantage of sex and recombination. Genet Res 55:199-221

Choleva L, Apostolou A, Rab P, Janko K (2008) Making it on their own: sperm-dependent hybrid fishes (Cobitis) switch the sexual host and expand beyond the ranges of their original sperm donors. Philos Trans R Soc Lond B Biol Sci 363:2911-2919. doi:10.1098/rstb.2008.0059

Constantz GD (1989) Reproductive biology of poeciliid fishes. In: Meffe GK, Snelson FF (eds) Ecology and evolution of livebearing fishes (Poeciliidae). Prentice Hall, New Jersey, pp 33-50

Cooper T, Lenski R, Elena S (2005) Parasites and mutational load: an experimental test of the pluralistic theory for the evolution of sex. Proc R Soc B Biol Sci 272:311-317

Dawley RM (1989) An introduction to unisexual vertebrates. In: Dawley RM, Bogart JP (eds) Evolution and ecology of unisexual vertebrates. Bulletin 466. New York State Museum, New York, pp 1-18

Gabriel W, Bürger R (2000) Fixation of clonal lineages under Muller's ratchet. Evol Int J Org Evol 54:1116-1125

Gabriel W, Lynch M, Bürger R (1993) Muller's ratchet and mutational meltdowns. Evol Int J Org Evol 47:1744-1757. doi:10.2307/2410218

Hamilton WD (1980) Sex versus non-sex versus parasite. Oikos 35:282-290. doi:10.2307/3544435

Hamilton WD, Axelrod R, Tanese R (1990) Sexual reproduction as an adaptation to resist parasites (a review). Proc Natl Acad Sci USA 87:3566-3573. doi:10.1073/pnas.87.9.3566

Heubel K (2004) Population ecology and sexual preferences in the mating complex of the unisexual Amazon molly Poecilia formosa (Girard, 1859). Dissertation, Universität Hamburg

Hubbs C (1964) Interactions between a bisexual fish species and its gynogenetic sexual parasite. Bull Tex Mem Mus 8:1-72

Killick S, Carlsson A, West S, Little T (2006) Testing the pluralist approach to sex: the influence of environment on synergistic interactions between mutation load and parasitism in Daphnia magna. J Evol Biol 19:1603-1611. doi:10.1111/j.1420-9101.2006.01123.x 
Kokko H, Heubel KU, Rankin DJ (2008) How populations persist when asexuality requires sex: the spatial dynamics of coping with sperm parasites. Proc R Soc B Biol Sci 275:817-825

Kondrashov AS (1982) Selection against harmful mutations in large asexual and sexual populations. Genet Res 40:325-332

Kondrashov AS (1988) Deleterious mutations and the evolution of sexual reproduction. Nature 336:435441. doi:10.1038/336435a0

Kondrashov AS (1993) Classification of hypotheses on the advantage of amphimixis. J Hered 84:372-387

Kondrashov AS (1999) Being too nice may be not too wise. J Evol Biol 12:1031. doi:10.1046/j.14209101.1999.00127.x

Kondrashov AS, Houle D (1994) Genotype-environment interactions and the estimation of the genomic mutation rate in Drosophila melanogaster. Proc R Soc B Biol Sci 258:221-227

Ladle RJ (1992) Parasites and sex: catching the Red Queen. Trends Ecol Evol 7:405-408. doi:10.1016/01695347(92)90021-3

Lampert KP, Schartl M (2008) The origin and evolution of a unisexual hybrid: Poecilia formosa. Philos Trans R Soc Lond B Biol Sci 363:2901-2909. doi:10.1098/rstb.2008.0040

Lampert KP, Lamatsch DK, Epplen JT, Schartl M (2005) Evidence for a monophyletic origin of triploid clones of the Amazon molly, Poecilia formosa. Evol Int J Org Evol 59:881-889

Lively CM (1989) Adaptation by a parasitic trematode to local populations of its snail host. Evol Int J Org Evol 43:1663-1671. doi:10.2307/2409382

Lively CM, Lloyd DG (1990) The cost of biparental sex under individual selection. Am Nat 135:489-500. doi: $10.1086 / 285058$

Lively CM, Craddock C, Vrijenhoek RC (1990) Red Queen hypothesis supported by parasitism in sexual and clonal fish. Nature 344:864-867. doi:10.1038/344864a0

Lively C, Lyons E, Peters A, Jokela J (1998) Environmental stress and the maintenance of sex in a freshwater snail. Evol Int J Org Evol 52:1482-1486. doi:10.2307/2411317

Loewe L, Lamatsch DK (2008) Quantifying the threat of extinction from Muller's ratchet in the diploid Amazon molly (Poecilia formosa). BMC Evol Biol 8:88. doi:10.1186/1471-2148-8-88

Maynard Smith J (1978) The evolution of sex. Cambridge University Press, Cambridge

Niemeitz A, Kreutzfeldt R, Schartl M, Schlupp I (2002) Male mating behaviour of a molly, Poecilia latipunctata: a third host for the sperm-dependent Amazon molly, Poecilia formosa. Acta Ethol 5:4549. doi:10.1007/s10211-002-0065-2

Riesch R, Schlupp I, Plath M (2008) Female sperm limitation in natural populations of a sexual/asexual mating complex (Poecilia latipinna, Poecilia formosa). Biol Lett 4:266-269. doi:10.1098/ rsbl.2008.0019

Ryan MJ, Dries LA, Batra P, Hillis DM (1996) Male mate preferences in a gynogenetic species complex of Amazon mollies. Anim Behav 52:1225-1236. doi:10.1006/anbe.1996.0270

Salathé M, Kouyos RD, Bonhoeffer S (2008) The state of affairs in the kingdom of the Red Queen. Trends Ecol Evol 23:439-445. doi:10.1016/j.tree.2008.04.010

Schartl M, Wilde B, Schlupp I, Parzefall J (1995) Evolutionary origin of a parthenoform, the Amazon molly Poecilia formosa, on the basis of a molecular genealogy. Evol Int J Org Evol 49:827-835. doi:10.2307/ 2410406

Schlupp I (2005) The evolutionary ecology of gynogenesis. Annu Rev Ecol Evol Syst 36:399-417. doi:10.1146/annurev.ecolsys.36.102003.152629

Schlupp I, Ryan MJ (1996) Mixed-species shoals and the maintenance of a sexual-asexual mating system in mollies. Anim Behav 52:885-890. doi:10.1006/anbe.1996.0236

Schories S, Lampert KP, Lamatsch DK, Garcia de Leon FJ, Schartl M (2007) Analysis of a possible independent origin of triploid P. formosa outside of the Rio Purification river system. Front Zool 4:13. doi:10.1186/1742-9994-4-13

Semlitsch RD, Hotz H, Guex G-D (1997) Competition among tadpoles of coexisting hemiclones of hybridogenetic Rana esculenta: support for the Frozen Niche Variation model. Evol Int J Org Evol 51:1249-1261. doi:10.2307/2411054

Tobler M, Schlupp I (2005) Parasites in sexual and asexual mollies (Poecilia, Poeciliidae, Teleostei): a case for the Red Queen? Biol Lett 1:166-168. doi:10.1098/rsbl.2005.0305

Tobler M, Schlupp I (2008) Expanding the horizon: the Red Queen and potential alternatives. Can J Zool 86:765-773. doi:10.1139/Z08-056

Tobler M, Wahli T, Schlupp I (2005) Comparison of parasite communities in native and introduced populations of sexual and asexual mollies of the genus Poecilia. J Fish Biol 67:1072-1082. doi:10.1111/ j.0022-1112.2005.00810.x

Vrijenhoek RC (1979) Factors affecting clonal diversity and coexistence. Am Zool 19:787-789 
Vrijenhoek RC (1994) Unisexual fish: model systems for studying ecology and evolution. Annu Rev Ecol Syst 25:71-96. doi:10.1146/annurev.es.25.110194.000443

Vrijenhoek RC, Pfeiler E (1997) Differential survival of sexual and asexual Poeciliopsis during environmental stress. Evol Int J Org Evol 51:1593-1600. doi:10.2307/2411211

West SA, Lively CM, Read AF (1999) A pluralist approach to sex and recombination. J Evol Biol 12:10031012. doi:10.1046/j.1420-9101.1999.00119.x

Wetherington JD, Schenck RA, Vrijenhoek RC (1989) The origins and ecological success of unisexual Poeciliopsis: the frozen niche-variation modell. In: Meffe GK, Snelson FF (eds) Ecology and evolution of lifebearing fishes (Poeciliidae). Prentice Hall, New Jersey, pp 259-275

Wolfe L (1993) Inbreeding depression in Hydrophyllum appendiculatum: role of maternal effects, crowding, and parental mating history. Evol Int J Org Evol 47:374-386. doi:10.2307/2410058 